\title{
Experiments with Face Recognition Using a Novel Approach Based on CVQ Technique
}

\author{
Arman Mehrbakhsh $^{1}$ and Alireza Khalilian ${ }^{2}$ \\ ${ }^{1}$ Sama Technical and Vocational Training College, Islamic Azad University \\ Andisheh Branch, Andisheh, Tehran, Iran \\ ${ }^{2}$ School of Computer Engineering, Iran University of Science and Technology, Tehran, Iran \\ ar_mehr@damavandiau.ac.ir, khalilian@comp.iust.ac.ir
}

\begin{abstract}
Face recognition techniques attempt to identify faces according to the patterns of mouth, lip, eyes and so on. However, the effectiveness of existing approaches degrades in presence of uncontrolled conditions such as variations of background light and image sizes. To deal with this problem, we propose a novel approach based on Classified Vector Quantization (CVQ) technique. The new approach divides images into some blocks and each block is classified into several patterns. Then, the Vector Quantization (VQ) technique is applied on the vectors of each pattern. In order to evaluate our approach, we have conducted a family of experiments on some standard image databases, MIT, YALE, and AR. The results demonstrate that the new approach is steadily capable of identifying faces in different situations.
\end{abstract}

Keywords: Face Recognition, Classified Vector Quantization (CVQ), Vector Quantization (VQ).

\section{Introduction}

Currently, due to the increment of violence and crime rate, the usage of systems to establish security and safety is a critical requirement to the current human's life [1]. To deal with this requirement, face recognition of convicts is considered as the major purpose in development of such systems [6]. In addition, since the events of September 11, the process of development and implementation of automated biometric systems have been noticeably increased [6, 7]. Moreover, there are many practical situations in which we need to the techniques of pattern recognition, and in particular face recognition. The wide range usage of these techniques in commercial and law enforcement applications, such as biometric authentication [7], video surveillance [39] and information security [1] has made it a popular and significant area of research. Nowadays, we are capable to identify people through automatic recognition techniques based on physiological and characteristic behaviours, such as finger print [28, 29], iris [2, 3, 4], ear [5], vein face [27], and so on. Although many techniques have been proposed for pattern recognition [30], especially face recognition [31, 32, 33], and a number of studies [34, 35, 36] have been performed in the literature to evaluate and compare the existing techniques, there is still a long road ahead to achieve optimal approaches. 
There are various natural characteristics in human's body such as finger print, ear, iris, and similar other parts whose structure are unique for each human [7]. The uniqueness of the structure for the mentioned body features can be used to recognize and to distinguish people from each other [37]. Ear recognition [5, 38] is a new class of biometrics that has certain advantages over most of the established biometrics; it has a rich and stable structure that is preserved from birth into the old ages [5]. Iris recognition $[2,4,9,8]$ is one of the most accurate biometric systems when a high level of security is required. Therefore, designing such systems has attracted the attention of a large number of researches [2, 4, 8, 9]. Unlike of other biometric methods, recognition based on human's face does not have any reliable and dependable mechanism that controls any undesirable conditions in the image of the face. These conditions include (1) changing the position of the face, (2) changing the size of the image, (3) the lack of sufficient light, etc. In ear recognition method, we need a half face image of each person. Hence, it is not an appropriate technique for facial expression recognition [5, 38]. In iris recognition method, by closing the eyes, the recognition process fails and we still need a reliable control mechanism. In addition, if the recognition relies only on the geometric structure of face $[25,26]$, it will also need a reliable control mechanism because face in various angles loses its potential to be recognized properly [10].

In this paper, we propose a novel approach for face recognition based on Classified Vector Quantization (CVQ) [17, 18, 23, 24] technique. At first step, this approach divides the face image into some blocks, converts each block to a vector, and classifies the vectors into some predefined patterns. These patterns are the major and common curves on the faces of different people. At second step, it uses Vector Quantization (VQ) $[11,13,14,15,16,19]$ on the classified vectors of each pattern. The selection of the predefined patters significantly affects the performance of this approach. To evaluate the functionality of the proposed approach, we have conducted experiments on some standard image databases used in the experimental studies in the literature. The overall results showed that the proposed approach is capable to recognize images with an appropriate recognition rate.

The remainder of this paper is organized as follows. In Section 2, we introduce the face recognition problem. Vector quantization techniques and classified vector quantization technique are described in Sections 3 and 4 respectively. In Section 5 the proposed approach is presented. The experimental setup and the obtained results are discussed in Sections 6. Finally, some concluding remarks are outlined in Section 7.

\section{Face Recognition}

Face recognition has been used in different fields of security and protection, namely automated crowd surveillance [39], access control [6], identification of convicts [40], face reconstruction $[43,44,45]$ and so on. Thus, it has been one of the challenging issues in the last decade and it is a necessity in our current life.

In computer vision, there are two important methods whose purpose is face recognition. The first group is holistic appearance base methods, including PCA [46, 47], and LDA [48, 49]. In these methods, a facial image is considered as an instance 
in $\mathrm{N}$ dimensional feature space, where $\mathrm{N}$ is the number of pixels in the image. The second group is local facial based methods such as elastic bunch graph matching. In these methods, a set of orthogonal basis vectors, that maximize the variance of facial image data, are obtained by Eigen decomposition of the scatter matrix of facial images. Combination of the two above methods, that are named hybrid methods, can be used for face recognition purpose.

Face recognition algorithms have focused on some unique properties of face [7] such as iris, ear, skin, etc. Among the different components of face, brow is the most important for face recognition purpose. According to the existing studies $[16,17,18$, 19], all of the face features or components do not have equal effect in the effectiveness of face recognition process. Most of the experimental results and researches [37] showed that in the field of face recognition, nose and mouth are more important than eyes. Although brows have equal effect in face recognition as compared with other face components, less attention has been considered toward the effects of brows against the other face features [11]. Thus, an algorithm that utilizes all of these features together would perform better than existing ones. The proposed method in this paper takes different features of the face into account to improve the efficacy of face recognition.

\section{Vector Quantization}

The Vector Quantization (VQ) technique [22] was first developed for image processing to compress the images [13, 14]. Codebook is a $2 \mathrm{D}$ array which is initialized randomly by the vectors of some of the training images. The number of columns in the codebook depends upon the size of the blocks of image and is usually considered as 9 or 16. In the decompressing phase, each code word by index $i$ is replaced by the code word with index $i$ in the codebook. One of the benefits of this technique is its low computational complexity. However, it is considered as a lossy technique. The CVQ [17, 18, 23, 24] and TSVQ [41, 42] are two major extensions of this technique.

When applying the VQ technique in face recognition, each block of the original image is identified with an index (number of the nearest code word in codebook). The response time is an important factor in real time applications [15] and VQ-based recognition provides reasonable response time in these situations.

In Vector Quantization (VQ) technique, the original image is partitioned into several blocks with the size of $\mathrm{n} \times \mathrm{m}$. Then, then each block is arranged in the form of a vector using the row major or the column major method. In the next step, the codebook is updated using the obtained vectors of the image. Each row of this matrix is called a code word. Next, the difference between each vector of the original image and each code word in the codebook is computed by using of Euclidean distance in Equation (1):

$$
D\left(B_{i}, C_{j}\right)=\sqrt{\sum_{k=1}^{n}\left(B_{i, k}-C_{j, k}\right)^{2}}
$$


In this equation, $B_{i}$ is a vector from the original image, $C_{j}$ is a code word from the codebook, $B_{i, k}$ is the $k$ th element of the $i$ th vector, and $C_{j, k}$ is the $k$ th element of the $j$ th code word. By this computation, the nearest code word to each vector with respect to the Euclidean distance is found. Finally, code words are updated by the centroid of all training vectors, which were mapped during coding [11]. Finding the optimized codebook is the major goal in VQ technique. It has been shown [12] that the design of VQ is optimal. This design is illustrated in Figure 1.

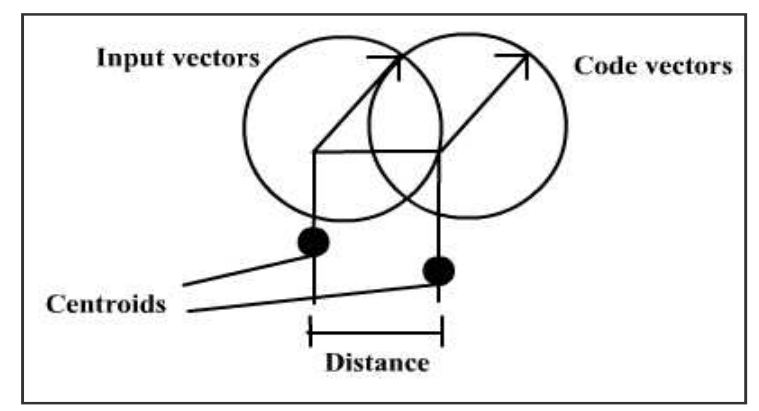

Fig. 1. Optimality of VQ design

\section{Classified Vector Quantization}

Edge is a very significant feature perceptually in an image. In our proposed method, we have used edge information for face recognition. Each coding technique that preserves the edge information is preferable because it has been proved [50] that the human's eye can recognize objects through their edges and this is adapted in image processing methods. The Classified Vector Quantization (CVQ) technique has been proved [17] to be an efficient technique for lossy image compression at low bit rates. CVQ technique can be used to reduce the computational complexity of VQ technique [11]. In this technique, each input vector is classified into a class. Then VQ is applied on vectors of each class [24]. Using this technique, there would be two indexes for each input vector: One for specifying the number of class and another for specifying the index of the nearest code word in codebook. This idea is shown in Figure 2. With CVQ technique, when applied to the face recognition, input vectors can be partitioned into some predefined patterns and then quantization is used for all vectors of each pattern. Since CVQ technique classifies the vectors in several classes, it is more precise than VQ.

\section{The Proposed Approach}

VQ technique can be very reliable in face recognition based on human's skin because of the correlation of human's skin. But the main problem is when the number of training vectors of images is increased, VQ cannot distinguish among vectors because 
many of the vectors happen to be similar and the differences can be hardly observed. As a result, the efficiency of this technique degrades and it is the only limitation in the usage of this technique. Now, each block of the test image is indexed with the number of a pattern. When all of the image blocks were indexed, VQ technique is applied over all vectors that were classified for each pattern.

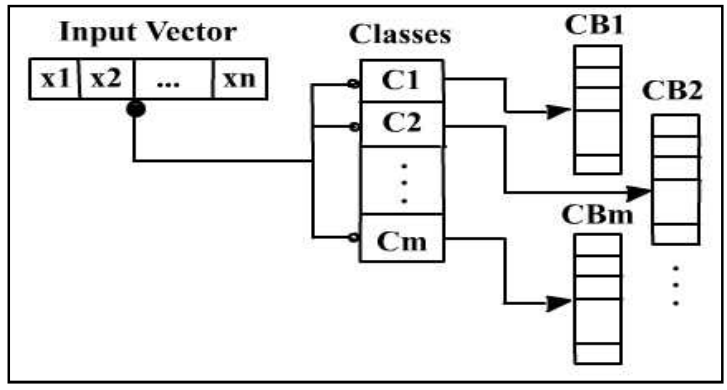

Fig. 2. A schematic of CVQ design

In order to deal with the issues of the VQ technique, CVQ can be applied. In our proposed approach, each block of the original image, the human's face, is compared with several predefined patterns. These patterns are defined according to the curves that are most seen in faces of each human. They can be extracted from a random number of faces using a pattern recognition approach like neural network. Figure 3 depicts some samples of patterns.

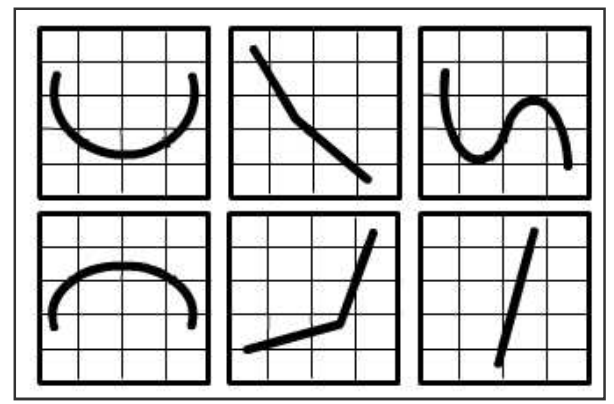

Fig. 3. Some samples of patterns

Several studies $[11,20,21]$ have shown that different features and components of the face can have unequal and various effects to the face recognition. Hence, taking all features of the face together into account has much potential for a face recognition technique to lead to better results. As a result, edge detection in face and also skin correlation can be considered together to enhance the face recognition process and improve the results. The Classified VQ technique enables us to consider the mentioned two features of the face simultaneously. This capability exposes the importance of CVQ technique in face recognition. 
According to the above descriptions, we can present our method in the following steps:

1. The image is cropped to fit in the desired size.

2. The image is converted to the grayscale.

3. We need to detect the edges of the faces related to each original image. For example, Sobel filter can be used for this purpose.

4. According to the previous step, we can classify each block of the original image to one of the predefined patterns. Suppose that all blocks have been classified into classes $C_{1}$ to $C_{n}$. Each class corresponds to a certain pattern. So, there would be a number for each block between 1 to $n$, so that it can describe the class number of the block.

5. After that, we can do vector quantization (VQ) technique on all original blocks which have the same class number. Note that there is a code book for each of the $n$ classes of patterns.

\section{Experimental Studies}

\subsection{VQ Results}

In order to implement the VQ technique to be applied in face recognition, we have used a low pass filter (2D- Moving average with mask 9×9) on each of face image at the first step. The images were selected from the standard image databases, AR [51], MIT [52], and ORL [53]. It can remove the noises of image and is able to detect the components of the face. Then, we applied a code book with 128 code vectors. Next, we tested the code books of different sizes and we found that code book with size 128 is the optimal.

Finally, for each training image, we created a histogram based on the number of similar vectors of each code word. Figure 4 shows a sample histogram for a face image. As a result, we will have a histogram for each of the training images. In order to find the output image, the histogram of input image and existing images in the database have been compared and the closest histogram was found.

We implemented this method using Delphi language, on standard image databases such as ORL, AR, Yale using a PC system with CPU $2.19 \mathrm{GHz}$ and $1.87 \mathrm{~GB}$ of Ram. We observed the recognition rate between $95 \%$ and $97 \%$ (depending on the size of used mask). In this experiment, Error Recognition Rate (ERR) has been measured to $2.6 \%$.

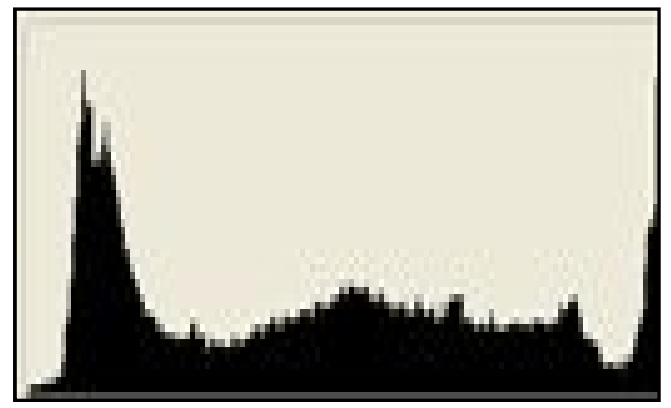

Fig. 4. A sample of face histogram obtained with the proposed approach 


\subsection{CVQ Results}

Observing the results obtained from the implementation of CVQ technique demonstrates that it has much potential for face recognition at high accuracy. As mentioned before, VQ is a robust technique for face recognition, especially in real time systems. However, when the number of input vectors of images is increased, it is no longer beneficial. Nevertheless, CVQ classifies the vectors into some patterns and then VQ can be used for vectors of each class. Moreover, by using this technique, we can utilize the two unique characteristics in human face, geometric structure of the face, and skin correlations. Hence, recognition rate is improved in comparison with other existing face recognition methods. The proposed method was implemented and tested using MIT-CBCL, AR, Yale and ORL image databases. At the first step, the faces in images are cropped. Then a filter such as Sobel filter is applied on each cropped face image in order to detect edges in faces. This enables us to classify each block of the size $n \times n$ in each of predefined patterns. Finally, VQ technique is used for all the blocks of the same pattern. We have implemented this method using the Java language, on a system with CPU $2.4 \mathrm{GHz}$ and $512 \mathrm{MB}$ of Ram. The recognition rate has been observed to be between $93 \%$ and $100 \%$ (depending on the type of database). Table 1 shows the recognition rate in some major face recognition methods and that of our proposed approach with respect to MIT image database.

Table 1. Recognition rate of some face recognition method along with our proposed approach

\begin{tabular}{cc}
\hline Method & Percent of Recognition Rate \\
\hline Global PZM & 82.91 \\
AWPPZMA & 92.31 \\
Eigen face $(\mathrm{k}=20)$ & 55.56 \\
Eigen face $(\mathrm{k}=60)$ & 69.23 \\
Eigen face $(\mathrm{k}=117)$ & 76.07 \\
Modular PCA $(\mathrm{k}=64)$ & 76.06 \\
Our method & 93 \\
\hline
\end{tabular}

The required time for pre-processing has been measured as $12 \mathrm{msec}$ and for recognition it has been measured at most $25 \mathrm{msec}$. Therefore, the total time would be $37 \mathrm{msec}$. The reason why we could achieve small processing time is that the proposed algorithm avoids from complex calculations. This will consequently improve the overall performance of the recognition with higher accuracy. We achieved the recognition rate of 45.83 and 37 percent for YALE and AR database images. For YALE database, there are 24 image groups, each group for an individual person. In each group, we have selected 5 images for training. In AR image database, there are images of different people in the same position and state. We have selected one image for each of the 65 persons. Then, we selected another image of each person to test such that it would be the same for all persons.

\section{Conclusions}

In this paper, a new face recognition approach based on Classified Vector Quantization (CVQ) has been proposed. We extended the concept of this technique in 
order to classify components of face images into some patterns and then we used Vector Quantization in order to create a code book for each pattern. In Comparison with other methods, our algorithm can be implemented without using hard mathematical computations.

\section{References}

1. Zhao, W., Chellappa, R., Phillips, P.J., Rosenfeld, A.: Face recognition: A literature survey. ACM Comput. Surv. 35(4), 399-458 (2003)

2. Estudillo-Romero, A., Escalante-Ramirez, B.: The Hermite Transform: An Alternative Image Representation Model for Iris Recognition. In: Ruiz-Shulcloper, J., Kropatsch, W.G. (eds.) CIARP 2008. LNCS, vol. 5197, pp. 86-93. Springer, Heidelberg (2008)

3. Bevilacqua, V., Cariello, L., Carro, G., Daleno, D., Mastronardi, G.: A face recognition system based on Pseudo 2D HMM applied to neural network coefficients. Soft Comput. 12(7), 615-621 (2008)

4. Roy, K., Bhattacharya, P.: Optimal Features Subset Selection Using Genetic Algorithms for Iris Recognition. In: Campilho, A., Kamel, M.S. (eds.) ICIAR 2008. LNCS, vol. 5112, pp. 894-904. Springer, Heidelberg (2008)

5. Attarchi, S., Nosrati, M.S., Faez, K.: A New Ear Recognition Approach for Personal Identification. In: Huang, D.-S., Wunsch II, D.C., Levine, D.S., Jo, K.-H. (eds.) ICIC 2008. LNCS, vol. 5226, pp. 356-363. Springer, Heidelberg (2008)

6. Butavicius, M., Mount, C., MacLeod, V., Vast, R., Graves, I., Sunde, J.: An Experiment on Human Face Recognition Performance for Access Control. In: Lovrek, I., Howlett, R.J., Jain, L.C. (eds.) KES 2008, Part I. LNCS (LNAI), vol. 5177, pp. 141-148. Springer, Heidelberg (2008)

7. Blackburn, T., Butavicius, M., Graves, I., Hemming, D., Ivancevic, V., Johnson, R., Kaine, A., McLindin, B., Meaney, K., Smith, B., Sunde, J.: Biometrics Technology Review, DSTO Systems Science Laboratory, Australia (2002)

8. Lim, S., Lee, K., Byeon, O., Kim, T.: Efficient Iris Recognition through Improvement of Feature Vector and Classifier. ETRI Journal 23(2), 61-70 (2001)

9. Ma, L., Tan, T., Wang, Y., Zhang, D.: Efficient iris recognition by characterizing key local variations. IEEE Trans. Image Proc. 13(6), 739-750 (2004)

10. Lu, Y.Z.: A Novel Face Recognition Algorithm for Distinguishing Faces with Various Angles. International Journal of Automation and Computing 5(2), 193-197 (2008)

11. Gersho, A., Gray, R.M.: Vector Quantization and Signal Compression. Kluwer Academic Publishers, Norwell (1991)

12. Jushi, M.A.: Digital image processing An algorithmic approach. Prentice-Hall Publishing (2006)

13. Hsieh, Y.P., Chang, C.C., Liu, L.J.: A two-codebook combination and three-phase block matching based image-hiding scheme with high embedding capacity. Pattern Recogn. 41(10), 3104-3113 (2008)

14. Kekre, H.B., Sarode, T.K.: Fast codevector search algorithm for 3-D Vector Quantized codebook. International Journal of Computer and Information Science and Engineering, 235-239 (2008)

15. Chang, C., Wu, W.: Fast plannar-oriented ripple search algorithm for Hyperspace VQ codebook. IEEE Trans. Image Proc. 16(6), 1538-1547 (2007)

16. Ramamurthi, B., Gersho, A.: Classified Vector Quantization of images. IEEE Trans. Communucations 34(11), 1105-1115 (1986) 
17. Quweither, M.K., Farison, J.B.: Classified Vector Quantization using Principal components. Electronics Letters 34(6), 538-540 (1998)

18. Kim, J.W., Lee, S.U.: A transform domain Classified vector Quantizer for image coding. IEEE Trans. Circuits and Systems for Video Technology 2(1), 3-14 (1992)

19. Kim, S., Lee, S.U.: Image Vector Quantizer based on a classification in the DCT domain. IEEE Trans. Communications 39(4), 549-556 (1991)

20. Young, A.W., Hay, D.C., McWeeny, K.H., Flude, B.M., Ellis, A.W.: Matching familiar and unfamiliar faces on internal and external features. Perception 14(6), 737-746 (1985)

21. Davies, G., Ellis, H., Shepherd, J.: Cue saliency in faces as assessed by the "Photofit" technique. Perception 6(3), 263-269 (1977)

22. Nasrabadi, N., King, R.: Image coding using vector quantization: A review. IEEE Trans. Communications 36(8), 957-971 (1988)

23. Tseng, H., Chang, C.: A Very Low Bit Rate Image Compressor Using Transformed Classified Vector Quantization. Informatica, 335-342 (2005)

24. Kim, J.W., Lee, S.U.: A transform domain classified vector quantizer for image coding. IEEE Trans. Circuits and Systems for Video Technology 2(1), 3-14 (1992)

25. Kanade, T.: Picture processing by computer complex and recognition of human faces. Ph.D. dissertation (1973)

26. Brunelli, R., Poggio, T.: Face recognition through geometrical features. In: European Conference on Computer Vision (1992)

27. Heenaye-Mamode Khan, M., Subramanian, R.K., Ali Mamode Khan, N.: Representation of Hand Dorsal Vein Features Using a Low Dimensional Representation Integrating Cholesky Decomposition. In: 2nd International Congress on Image and Signal Processing, CISP 2009 (2009)

28. Hong, L., Jian, A., Pankanti, S., Bolle, R.: Fingerprint enhancement. In: Proceedings 3rd IEEE Workshop on Applications of Computer Vision, WACV 1996 (1996)

29. Ito, K., Morita, A., Aoki, T., Higuchi, T., Nakajima, H., Kobayashi, K.: A fingerprint recognition algorithm using phase-based image matching for low-quality fingerprints. In: IEEE International Conference on Image Processing, ICIP 2005 (2005)

30. Jain, A.K., Duin, R.P.W., Mao, J.: Statistical Pattern Recognition: A Review. IEEE Transactions on Pattern ANALYSIS and Machine Intelligence 22(1) (January 2000)

31. Guo, G.-D., Zhang, H.-J.: Boosting for fast face recognition. In: Proceedings of the IEEE ICCV Workshop (2001)

32. Watanabe, E., Kodate, K.: Ultra-fast facial recognition system based on VanderLugt Correlator. In: CLEO/Pacific Rim 2003 - The 5th Pacific Rim Conference on Lasers and Electro-Optics (2003)

33. Momeni, H., Sadeghi, M.T., Abutalebi, H.R.: Fast face recognition using a combination of image pyramid and hierarchical clustering algorithms. In: International Conference on Wireless Communications \& Signal Processing, WCSP 2009 (2009)

34. Sani, M.M., Ishak, K.A., Samad, S.A.: Evaluation of face recognition system using Support Vector Machine. In: 2009 IEEE Student Conference on Research and Development, SCOReD (2009)

35. Phillips, P.: The FERET Evaluation Methodology for Face-Recognition Algorithms. IEEE Transaction 22 (2000)

36. Utsumi, Y., Iwai, Y., Yachida, M.: Performance Evaluation of Face Recognition in the Wavelet Domain. In: 2006 IEEE/RSJ International Conference on Intelligent Robots and Systems (2006) 
37. Dewi Agushinta, R., Suhendra, A., Madenda, S., Suryadi, H.S.: Face Component Extraction Using Segmentation Method on Face Recognition System. Journal of Emerging Trends in Computing and Information Sciences (2011)

38. Ali, M., Javed, M.Y., Basit, A.: Ear Recognition Using Wavelets. In: Proceedings of Image and Visioin Computing (2007)

39. Zhong, Z., Ding, N., Wu, X., Xu, Y.: Crowd surveillance using Markov Random Fields. In: IEEE International Conference on Automation and Logistics, ICAL 2008 (2008)

40. Daygman, J.G.: High Confidence Visual Recognition of persons by a test of Statistical Independence. IEEE Transaction (1993)

41. Makwana, M.V., Nandurbarkar, A.B., Joshi, S.M., Coll, L.E.: Image Compression Using Tree Structured Vector Quantization with Compact Codebook. In: International Conference on Computational Intelligence and Multimedia Applications (2007)

42. Chen, J.-Y., Bouman, C.A., Allebach, J.P.: Fast image database search using treestructured VQ. In: Proceedings of the International Conference on Image Processing (1997)

43. Park, U., Jain, A.K.: 3D Face Reconstruction from Stereo Video. In: The 3rd Canadian Conference on Computer and Robot Vision (2006)

44. Zhao, M., Chua, T.-S., Sim, T.: Morphable Face Reconstruction with Multiple Images. In: Proceedings of the 7th International Conference on Automatic Face and Gesture Recognition (2006)

45. Wang, S.-F., Lai, S.-H.: Reconstructing 3D Face Model with Associated Expression Deformation from a Single Face Image via Constructing a Low-Dimensional Expression Deformation Manifold. IEEE Transactions on Pattern Analysis and Machine Intelligence (2011)

46. Weingessel, A., Hornik, K.: Local PCA algorithms. IEEE Transactions on Neural Networks (2000)

47. Diamantaras, K.I., Kung, S.Y.: Principal Component Neural Networks: Theory and Applications. John Wiley \& Sons, Inc. (1996)

48. Lu, J., Plataniotis, K.N., Venetsanopoulos, A.N.: Face Recognition Using LDA Based Algorithms. IEEE Transactions on Neural Networks (2002)

49. Martinez, A.M., Kak, A.C.: PCA versus LDA. IEEE Transactions on Pattern Analysis and Machine Intelligence (2001)

50. Lindeberg, T.: Edge Detection and Ridge Detection with Automatic Scale Selection. In: 1996 IEEE Computer Society Conference on Computer Vision and Pattern Recognition (1996)

51. Martinez, A.M., Benavente, R.: The AR Face Database. CVC Technical Report \#24 (1998)

52. Pissarenko, D.: Face databases (2003)

53. ORL face database, http://www.uk.research.att.com/facedatabase.html 